\title{
P2X7, NMDA and BDNF receptors converge on GSK3 phosphorylation and cooperate to promote survival in cerebellar granule neurons
}

\author{
Felipe Ortega • Raquel Pérez-Sen • Verónica Morente • \\ Esmerilda G. Delicado • Maria Teresa Miras-Portugal
}

Received: 21 October 2009/Revised: 22 December 2009/Accepted: 18 January 2010/Published online: 10 February 2010

(C) The Author(s) 2010. This article is published with open access at Springerlink.com

\begin{abstract}
Glycogen synthase kinase-3 (GSK3) is a key player in the regulation of neuronal survival. Herein, we report evidence of an interaction between $\mathrm{P} 2 \mathrm{X} 7$ receptors with NMDA and BDNF receptors at the level of GSK3 signalling and neuroprotection. The activation of these receptors in granule neurons led to a sustained pattern of GSK3 phosphorylation that was mainly PKC-dependent. BDNF was the most potent at inducing GSK3 phosphorylation, which was also dependent on PI3K. The P2X7 agonist, BzATP, exhibited additive effects with both NMDA and BDNF to rescue granule neurons from cell death induced by PI3K inhibition. This survival effect was mediated by the PKC-dependent GSK3 pathway. In addition, ERK1/2 proteins were also involved in BDNF protective effect. These results show the function of ATP in amplifying neuroprotective actions of glutamate and neurotrophins, and support the role of GSK3 as an important convergence point for these survival promoting factors in granule neurons.
\end{abstract}

Keywords GSK3 - Granule neurons - P2X7 ·

Nucleotide receptors · Neuroprotection · Survival factors · Nucleotides

F. Ortega and R. Pérez-Sen have equally contributed to this work.

Electronic supplementary material The online version of this article (doi:10.1007/s00018-010-0278-x) contains supplementary material, which is available to authorized users.

F. Ortega $\cdot$ R. Pérez-Sen $(\bowtie) \cdot$ V. Morente

E. G. Delicado - M. T. Miras-Portugal

Department of Biochemistry, Veterinary Faculty,

Complutense University of Madrid, Madrid, Spain

e-mail: rpsen@vet.ucm.es

\section{Introduction}

Cerebellar granule neurons provide an excellent model to study the cellular mechanisms underlying survival and neuronal maintenance [1]. The actions of several trophic factors and the intracellular signalling cascades that they trigger in order to promote neuronal survival is well documented, as for example IGF-I and BDNF [2]. Recently, in addition to these well known factors, purine and pyrimidine nucleotides have been characterised as important extracellular signaling molecules mediating different functions of physiological relevance in granule neurons. These neurons have been reported to co-express a great diversity of nucleotide receptors from the ionotropic $\mathrm{P} 2 \mathrm{X}$ and metabotropic P2Y families [3, 4]. Ionotropic P2X nucleotide receptors are ligand-operated channels which are coupled to the entry of extracellular calcium [5]. The metabotropic P2Y receptors are G-protein coupled receptors mainly associated with PLC and adenylate cyclase signalling [6]. In previous studies, functional calcium responses have been demonstrated at the level of rat cerebellar synaptic terminals and granule cell fibres for P2X1, P2X3, P2X4 and $\mathrm{P} 2 \mathrm{X} 7$ receptors [7]. In addition, in rat granule neurons in culture, $\mathrm{P}_{2} \mathrm{Y}_{1}$ receptors have also been shown to be coupled to increases in the intracellular calcium concentration [3]. Following calcium-related events, several of these receptors, such as $\mathrm{P}_{2} \mathrm{Y}_{1}, \mathrm{P} 2 \mathrm{X} 3$ and $\mathrm{P} 2 \mathrm{X} 7$, were also shown to induce CaMKII and synapsin I phosphorylation and subsequent glutamate release in rat granule neurons [8].

Recent studies have further investigated the intracellular signalling mechanisms triggered by nucleotide receptors and have demonstrated the coupling of $\mathrm{P}_{2} \mathrm{Y}_{13}$ and $\mathrm{P} 2 \mathrm{X} 7$ receptors to GSK3 phosphorylation in rat cerebellar granule neurons. The activation of both these nucleotide 
receptors resulted in an increase in the phosphorylation levels of GSK3 at specific Ser residues that were associated with the inhibition of its catalytic activity [9]. However, these nucleotide receptors displayed different intracellular mechanisms to reach GSK3. P2 $\mathrm{Y}_{13}$ receptors were coupled to the well characterized PI3K/Akt dependent pathway, described for growth factors of the insulin family [10], resulting in the stabilization of the GSK3 substrate $\beta$-catenin and its translocation to the nucleus, where it functions as a transcriptional regulator [11]. Nevertheless, P2X7-induced GSK3 phosphorylation occurred through a PKC-dependent pathway. This signalling mechanism was responsible for the protective effect that the $\mathrm{P} 2 \mathrm{X} 7$ nucleotide agonist, BzATP, exhibited against cell death induced by the inhibition of the PI3K/Akt survival route [12].

The role of GSK3 as a key regulator of the survival/ apoptosis balance in neurons is supported by a great deal of evidences [13]. Indeed, over-expression of this protein is linked to apoptotic cell death in several neuronal models [14]. Among different potential GSK3 targets, Bax, a protein of the intrinsic apoptotic cascade that is involved in mitochondrial pore formation, has been reported to become phosphorylated and translocated to mitochondria upon GSK3 activation in granule neurons [15]. In addition, GSK3 cooperates and contributes to apoptotic signalling, such as JNK activation, during trophic deprivation [16]. In this context, several treatments capable of rescuing granule neurons from low-potassium induced apoptosis, such as high potassium, IGF-I and cAMP, were described to converge on GSK3 inhibition [17]. What is common for these factors is that they employ the PI3K/Akt/GSK3-dependent pathway, and this is defined as the main survival route in neurons against apoptotic death induced by trophic support withdrawal [18]. Following the same line of evidence, the sole expression of the dominant negative GSK3 mutant is able to rescue cortical neurons from death induced by serum deprivation [19].

Taking into account the survival effect displayed by the activation of $\mathrm{P} 2 \mathrm{X} 7$ receptors in granule neurons, it is tempting to speculate that nucleotides could be functioning as priming molecules either complementing or potentiating the effects of other survival signals in granule neurons. In the first approach we focused on NMDA, as representative of activity dependent effects, and BDNF, as representative of trophic like actions. Although their survival promoting effects have been well established in this model, their signalling route to GSK3 has not been deeply characterized [20]. In the present study, we have found out that NMDA and BDNF receptors are also coupled to GSK3 phosphorylation in granule neurons through both PKC and PI3K/Akt dependent mechanisms. We also describe that they are able to use the PKC/GSK3 pathway to induce neuronal survival in conditions of PI3K impairment. In the case of BDNF,
ERK proteins are also involved. Additionally, we have also demonstrated that $\mathrm{P} 2 \mathrm{X} 7$ receptors cooperate with both NMDA and BDNF receptors in promoting neuronal survival.

\section{Materials and methods}

Culture of cerebellar granule neurons

All experiments carried out at the Universidad Complutense de Madrid followed the guidelines of the International Council for Laboratory Animal Science (ICLAS). Cerebellar cultures were performed according to the procedure described by Pons et al. [21]. Cerebella from Wistar rat pups (P7) were aseptically removed, and submitted to digestion with papain $100 \mathrm{U} / \mathrm{ml}$ (Worthington, Lake Wood, NJ) (previously activated in EBSS buffer containing of $5 \mathrm{mM}$ L-Cys, $2 \mathrm{mM}$ EDTA and $0.067 \mathrm{mM}$ B-mercaptoethanol), in the presence of $100 \mathrm{U} / \mathrm{ml}$ of DNase (Worthington, Lake Wood, NJ), $1 \mathrm{mM} \mathrm{CaCl} \mathrm{Cl}_{2}$ and $1 \mathrm{mM}$ $\mathrm{MgCl}_{2}$. The obtained cells were resuspended in neurobasal medium supplemented with B-27 (GIBCO BRL, Paisley, Renfrewshire, UK) and containing $21 \mathrm{mM} \mathrm{KCl}, 2 \mathrm{mM}$ glutamine, and antibiotics, $100 \mathrm{U} / \mathrm{ml}$ penicillin, $0.1 \mathrm{mg} / \mathrm{ml}$ streptomycin and $0.25 \mu \mathrm{g} / \mathrm{ml}$ amphotericin B (SigmaAldrich, St Louis, USA), and were plated onto glass coverslips or plastic Petri dishes (100 mm) (Falcon BectonDickinson Labware, Franklin Lakes, USA) precoated with $0.1 \mathrm{mg} / \mathrm{ml}$ poly-L-lysine (Biochrom, AG, Berlin) at a density of 200,000 cells $/ \mathrm{cm}^{2}$. They were maintained in a humidified incubator at $37^{\circ} \mathrm{C}$ in $5 \% \mathrm{CO}_{2}$. Ara-C $(10 \mu \mathrm{M})$ was added to avoid the proliferation of glial cells.

\section{Cell treatments}

Cultured cerebellar granule neurons were used at 911 DIV (days in vitro). In the experiments regarding GSK3 phosphorylation, prior to the stimulation of cells with the different effectors, cells were routinely washed for about $2 \mathrm{~h}$ at $37^{\circ} \mathrm{C}$ in a low potassium medium [Locke solution, in $\mathrm{mM}: \mathrm{NaCl}, 140 ; \mathrm{KCl}, 4.7 ; \mathrm{CaCl}_{2}, 2.5 ; \mathrm{KH}_{2} \mathrm{PO}_{4}, 1.2$; $\mathrm{MgSO}_{4}, 1.2$; glucose, 5.5; and HEPES (acid), 10; $\left.\mathrm{pH} 7.4\right]$ in order to lower the basal levels of GSK3 and ERK1/2 phosphorylation, which are normally elevated as a consequence of the high potassium medium. After this washing period, the medium was again replaced by normal Locke solution in the case of BDNF, or by Locke solution in the absence of $\mathrm{Mg}^{2+}$, in the case of BzATP and NMDA, and then the corresponding agents were added at the required concentrations for the required incubation times. $\mathrm{Mg}^{2+}$. free medium was used in order to enhance P2X7 and NMDA-mediated responses, as both receptors are known to 
be sensitive to the inhibition by divalent cations. BzATP and BDNF were assayed at maximal saturating concentrations of $300 \mu \mathrm{M}$ and $50 \mathrm{ng} / \mathrm{ml}$, respectively. Nevertheless, in the case of NMDA, a submaximal concentration of $50 \mu \mathrm{M}$ was preferred over $100 \mu \mathrm{M}$, in order to avoid possible excitotoxic actions.

Survival studies required long-term periods, and in this case, the treatments were applied in complete culture medium. BzATP, NMDA and BDNF were added $10 \mathrm{~min}$ before the addition of the PI3K inhibitor LY-294002. Cell viability was then evaluated $24 \mathrm{~h}$ later. When receptor antagonists and transducing inhibitors were employed, they were added 10 or 20 min before the agonists.

\section{Western blot experiments}

Cells were stimulated in the presence of the nucleotide agonists and IGF-I with different treatments for the required times. Stimulation was stopped by addition of lysis buffer that consisted of: $20 \mathrm{mM}$ MOPS, $50 \mathrm{mM} \mathrm{NaF}$, $40 \mathrm{mM} \beta$-glycerophosphate, $1 \mathrm{mM}$ sodium orthovanadate, $5 \mathrm{mM}$ EDTA, $2 \mathrm{mM}$ EGTA, 0.5\% Triton X-100, pH 7.2, $1 \mathrm{mM}$ PMSF and protease inhibitor cocktail (Complete, Roche). Protein determination of the cell extracts was performed and then mixed with sample buffer $4 \times(50 \%$ Glycerol, $125 \mathrm{mM}$ Tris $\mathrm{pH}$ 6.8, 4\% SDS, 1\% bromophenol blue, $5 \% \quad \beta$-mercaptoetanol, $\left.4,5 \% \quad \mathrm{H}_{2} \mathrm{O}\right)$. The samples ( $25 \mu \mathrm{g}$ protein) were heated at $99^{\circ} \mathrm{C}$ and aliquots were subjected to sodium dodecyl sulfate (SDS) gel electrophoresis (25 mM Tris, $200 \mathrm{mM}$ glycine, $0.1 \%$ SDS, $\mathrm{pH}$ 8.3) using $12 \%$ acrylamide gels. Immunotransference was performed on PVDF membranes (Amersham Biosciences Europe GmbH, Barcelona, Spain) (25 mM Tris, $192 \mathrm{mM}$ glycine, 20\% methanol). The TBS buffer (100 mM NaCl, $10 \mathrm{mM}$ Tris- $\mathrm{HCl}, \mathrm{pH} 7.5$ ) containing $1 \%$ (v/v) Tween-20 and $5 \%$ BSA was employed as a blocking medium and was used in subsequent incubations with the antibodies. Incubation with the antibodies was performed at the following dilutions: 1:750 for phospho-GSK3 $\alpha / \beta$ (Ser21/9), caspase3 , $\beta$-III tubulin (all of them from Cell Signalling Technology, Beverly, MA, USA), and 1:1,000 for total GSK3 (Biosource, Nivelles, Belgium), phospho-ERK1/2 (Thr202/ Tyr204), total ERK (Santa Cruz Biotechnologies Inc., Santa Cruz, CA, USA). Primary antibodies were detected with horseradish peroxidase-conjugated antibodies, 1:4,000 for anti-mouse (Santa Cruz Biotechnology Inc., Santa Cruz, CA) and 1:4,000 for anti-rabbit (Jackson ImmunoResearch Laboratories, West Grove, PA, USA), and visualized by the ECL method (kit Super Signal substrate Wester Blotting, from Amersham Biosciences Europe $\mathrm{GmbH}$, Barcelona, Spain). The chemiluminscence images were quantified by densitometry employing the Fluo-S Imager, Bio-Rad (Munich, Germany).
Cell viability assays

Neuronal survival was assayed by both LIVE/DEAD viability/cytotoxicity kit (Molecular Probes) and MTT assay. The LIVE/DEAD viability/cytotoxicity assay was applied after the corresponding treatment with LY-294002. This measures the number of live cells that have incorporated the green fluorescent calcein dye versus the number of dead cells, which have incorporated the red ethidium dye (ethidium homodimer-1). The immunofluorescence pictures were taken with a Nikon TE-200 microscope and a Kappa DX2 camera controlled by Kappa Image Base Control software.

In the MTT assay the mitochondrial function is assessed. This method employed MTT tetrasodium salt [3-(4,5dimethythiazol-2-yl)-2,5-diphenyl tetrazolium bromide] (Sigma), which was added to the cultures after the LY294002 treatment, at a final concentration of $0.5 \mathrm{mg} / \mathrm{ml}$, and was maintained for $2 \mathrm{~h}$ at $37^{\circ} \mathrm{C}$. Then, an equal volume of MTT solubilization solution (10\% Triton X-100 plus $0.1 \mathrm{~N} \mathrm{HCl}$ in anhydrous isopropanol) was added, following a brief incubation of $30 \mathrm{~min}$ at room temperature with orbital shaking. The samples were collected and measured spectrophotometrically at $570 \mathrm{~nm}$. Values were normalized with respect to that obtained from untreated cells, considered as $100 \%$ survival.

Statistical analysis

Data are represented as mean $\pm \mathrm{SD}$ of at least three independent experiments obtained from different cultures. Comparison between different treatments and controls were carried out using Dunnett's test, and comparisons between different samples were performed using Tukey's test. A probability of $<0.05$ was considered significant. Data were statistically significant at $* * *$ /\#\#\#P $<0.001$, $* * / \# \# P<0.01$ and $* / \# P<0.05$.

\section{Results}

BzATP, NMDA and BDNF are coupled to GSK3 phosphorylation in cerebellar granule neurons

Phosphorylation of GSK3 at $\operatorname{Ser}^{21}$ and $\operatorname{Ser}^{9}$ residues for $\alpha$ and $\beta$ isoforms, respectively, can be taken as indicative of its inhibition level, and can be evaluated with specific antibodies against the phorphorylated forms. In a previous work, the effect of several nucleotidic agonists on GSK3 phosphorylation was investigated, and among them, BzATP resulted to be the most potent, through the activation of the $\mathrm{P} 2 \mathrm{X} 7$ nucleotide receptor. We demonstrated that BzATP-induced GSK3 phosphorylation was 
dependent on extracellular calcium, and sensitive to P2X7 specific antagonists [12]. In the present study the effect of BzATP on GSK3 phosphorylation was compared to other relevant extracellular signals in granule neurons, such as glutamate and BDNF.

As can be seen in Fig. 1A, BzATP $(300 \mu \mathrm{M})$, NMDA $(50 \mu \mathrm{M})$ and BDNF $(50 \mathrm{ng} / \mathrm{ml})$ were able to induce significant increases in GSK3 phosphorylation over basal levels. They exhibited a similar time-dependent pattern, as GSK3 phosphorylation was sustained over $1-2 \mathrm{~h}$ of stimulation periods with the three stimuli. BDNF was the fastest and most potent signal, producing the highest levels of GSK3 phosphorylation at as early as $5 \mathrm{~min}$ of stimulation. This agrees with the rapid BDNF-mediated signalling reported in granule and hippocampal neurons [22]. In the case of NMDA and BzATP the increase percentages in GSK3 phosphorylation were in the same range, being always slightly higher for NMDA.

In subsequent experiments we analyzed in more detail the intracellular signalling mechanism of GSK3 phosphorylation induced by the three stimuli, employing inhibitors of different signal transducing proteins that can be upstream of GSK3. Taking into account that PI3K/Akt is very important in the signalling elicited by both NMDA and BDNF, this pathway was selectively blocked by the treatment with the specific inhibitor LY-294002. As expected, LY-294002 (50 $\mu \mathrm{M}, 20 \mathrm{~min})$ was able to abolish BDNF-induced increase in GSK3 phosphorylation, but only partially affected the BzATP and NMDA-mediated effects (Fig. 1b).

In accordance with the mechanism of action of TrkB receptors activated by BDNF in granule neurons, the pretreatment with Gö $6976(0.5 \mu \mathrm{M}, 20 \mathrm{~min})$, an inhibitor of the calcium-dependent PKC subtypes, eliminated BDNFmediated responses. PKC inhibition also blocked the BzATP-mediated increase in GSK3 phosphorylation, but a residual effect seemed to persist after NMDA stimulation. Finally, we assessed the role of the MAPK pathway in GSK3 regulation using U-0126 $(10 \mu \mathrm{M}, 20 \mathrm{~min})$, an inhibitor of MEK-1 that is upstream ERK1/2. These proteins did not affect the increase in GSK3 phosphorylation levels induced by BzATP, NMDA or BDNF (Fig. 1b).

To substantiate the calcium dependence of GSK3 inhibition displayed by the effectors, other strategies were tested. When extracellular calcium was chelated by a mixture of EGTA/Tris, BzATP and NMDA-induced GSK3 phosphorylation was totally abrogated, as expected for the ionotropic nature of the receptors, P2X7 and NMDA receptors. In addition, intracellular calcium quelation by BAPTA also abolished BDNF-mediated effect (Supplementary Fig. 1A). These results support the involvement of classical calcium-dependent PKC isoforms, probably $\operatorname{PKC} \alpha$ and $\operatorname{PKC} \beta 1$, which are the predominant subtypes
(A)
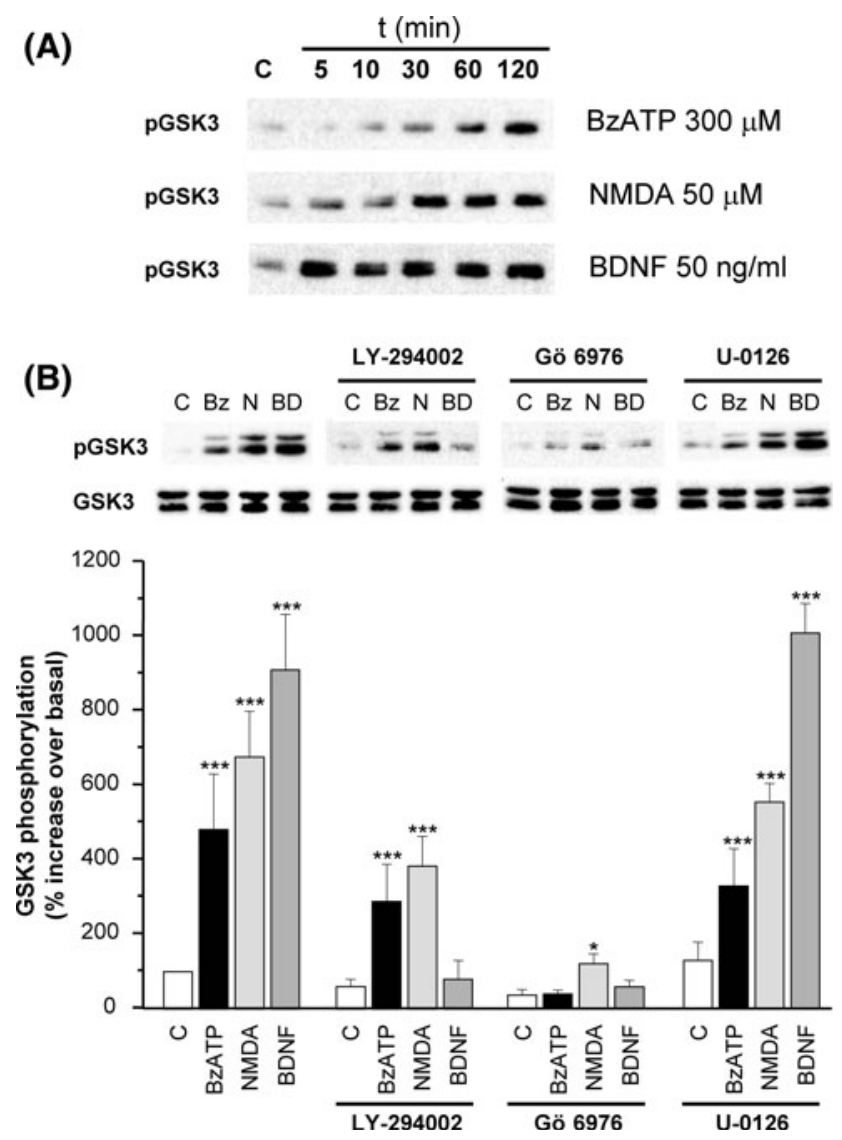

Fig. 1 GSK3 phosphorylation induced by BzATP, NMDA and BDNF in granule neurons. a Time-course of GSK3 phosphorylation. Granule cells were stimulated with $300 \mu \mathrm{M}$ BzATP, $50 \mu \mathrm{M}$ NMDA and $50 \mathrm{ng} / \mathrm{ml} \mathrm{BDNF}$, at different incubation times. b Effect of various signalling pathway inhibitors on GSK3 phosphorylation. Granule cells in culture were submitted to different treatments: $20 \mathrm{~min}$ incubation with signalling inhibitors, $50 \mu \mathrm{M}$ LY-294002, $0.5 \mu \mathrm{M}$ Gö 6976 and $10 \mu \mathrm{M} \mathrm{U}-0126$. Then cells were stimulated for $10 \mathrm{~min}$ with $300 \mu \mathrm{M} \mathrm{BzATP}, 50 \mu \mathrm{M}$ NMDA and $50 \mathrm{ng} / \mathrm{ml}$ BDNF in the continuous presence of these inhibitors. Then cells were harvested and phosphorylation of GSK3 was analysed by immunoblotting. Histograms represent the percentage increase with respect to nonstimulated cells (100\% control value), and were obtained by normalization of densitometric values of phospho-proteins with respect to total forms of GSK3. The blots correspond to representative experiments and values are the mean $\pm \mathrm{SD}$ of at least three experiments performed from different cultures. Data were analysed by Dunnet and Tukey tests, and were statistically significant at $* P<0.05$ and $* * * P<0.01$ when the effects were compared to the respective control for each condition (open bars in the absence stimulation)

expressed in cultured granule neurons at this stage of differentiation [23].

P2X7 and NMDA receptors converge on GSK3 phosphorylation in granule neurons

The above results indicate that some differences are clear among the factors analyzed with respect to the signalling 
(A)
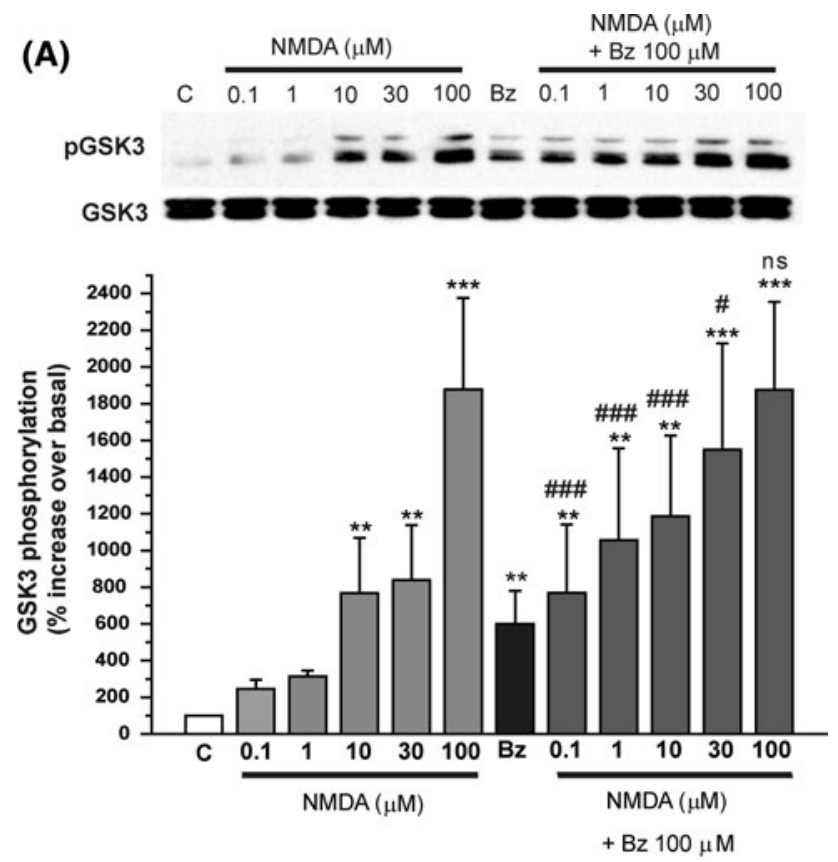

(B)
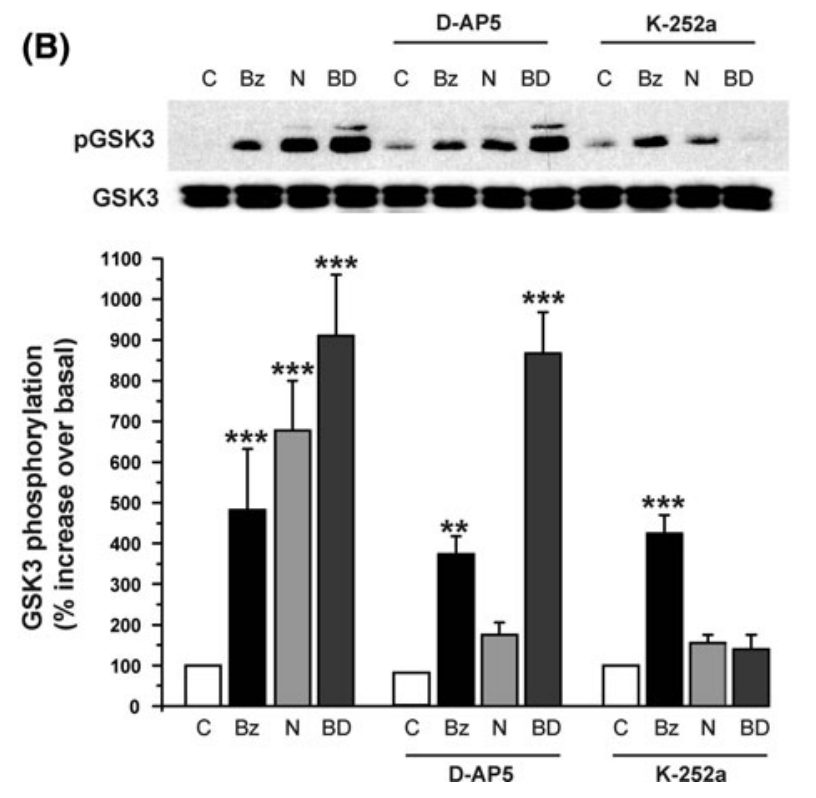

mechanisms reaching GSK3, which could account for a possibility of additive effects upon co-stimulation. Figure 2a shows the dose-response curve for NMDA-induced GSK3 phosphorylation performed in the absence and presence of co-stimulation with the nucleotide agonist BzATP. It can be observed that when suboptimal concentrations of both effectors were added together, the levels of GSK3 phosphorylation were higher with respect to that obtained with either BzATP or NMDA alone. This is in agreement with BzATP and NMDA converging on the same PKC/GSK3 dependent pathway. Similar studies carried out with BzATP and BDNF did not shown any cooperation on GKS3 phosphorylation, even using
Fig. 2 Convergence of BzATP and NMDA in GSK3 phosphorylation. a Effect of combined treatment of BzATP and NMDA on GSK3 phosphorylation. Granule neurons were stimulated for $10 \mathrm{~min}$ with different NMDA concentrations (from 0.1 to $100 \mu \mathrm{M}$ ) in the absence or presence of co-stimulation with $100 \mu \mathrm{M}$ BzATP concentration. b Effect of NMDA antagonist and kinase inhibitor of TrkB receptor.

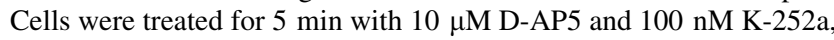
and then $300 \mu \mathrm{M}$ BzATP, $50 \mu \mathrm{M}$ NMDA and $50 \mathrm{ng} / \mathrm{ml}$ BDNF were added for additional $10 \mathrm{~min}$. Then cells were harvested and phosphorylation of GSK3 was analysed by immunoblotting, as described in "Material and methods". Data were obtained by normalization of densitometric values of phospho-GSK3 with respect to total GSK3. The blots correspond to representative experiments and values are the mean $\pm \mathrm{SD}$ of at least three experiments performed from different cultures. Data were analysed by Dunnet and Tukey tests, and were statistically significant at $* * * P<0.001$ and $* * P<0.01$ when the effects were compared to the controls (empty bars in the absence stimulation) (a, b), and at ${ }^{\# \#} P<0.001$, and ${ }^{\#} P<0.05$, when the values at each NMDA concentration was compared in the absence and presence of BzATP a. $B z$ BzATP, $N$ NMDA, $B D$ BDNF

submaximal BDNF concentrations $(0.5 \mathrm{ng} / \mathrm{ml})$ (Supplementary Fig. 2).

Therefore, it can be concluded that BzATP is potentiating the effect of NMDA on GSK3 phosphorylation in granule neurons. In order to assess the specificity of the effects, the NMDA specific receptor antagonist, D-AP5 $(10 \mu \mathrm{M})$, was employed, and as shown in Fig. $2 \mathrm{~b}$, this treatment significantly affected NMDA-mediated responses. In the case of the BDNF receptor, a kinase inhibitor of Trk type receptors was used, K-252a $(100 \mathrm{nM})$, which partially blocked the effect of NMDA, but not that of BzATP. These results confirm that, although Trk activity seems to partially contribute to NMDA-mediated response, it has no effect on BzATP response.

\section{BzATP cooperates with NMDA and BDNF}

in promoting granule cell survival against PI3K

pharmacological inhibition

The coupling of BzATP, NMDA and BDNF to GSK3 regulation led us to investigate the physiological meaning of this signalling mechanism. In fact, GSK3 has been reported to play a major role in cell death induced by trophic withdrawal, an apoptotic outcome that can be reproduced by the blockade of the PI3K/Akt axis. By using this approach, we have tested the possible neuroprotective role of these agents. In addition to BzATP, NMDA and BDNF were able to elicit survival in conditions of sustained PI3K inactivation with the inhibitor LY-294002 $(50 \mu \mathrm{M})$ (Fig. 3a, b). This indicated that, although NMDA and BDNF receptors are coupled to PI3K/Akt signalling, they can by-pass the PI3K/Akt axis and display alternative survival routes. NMDA resulted to be always more effective than BzATP and BDNF in promoting granule cell survival, as survival levels with NMDA reached about 
(A)

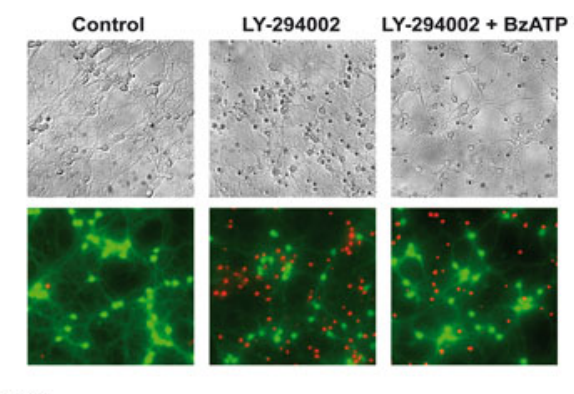

(B)
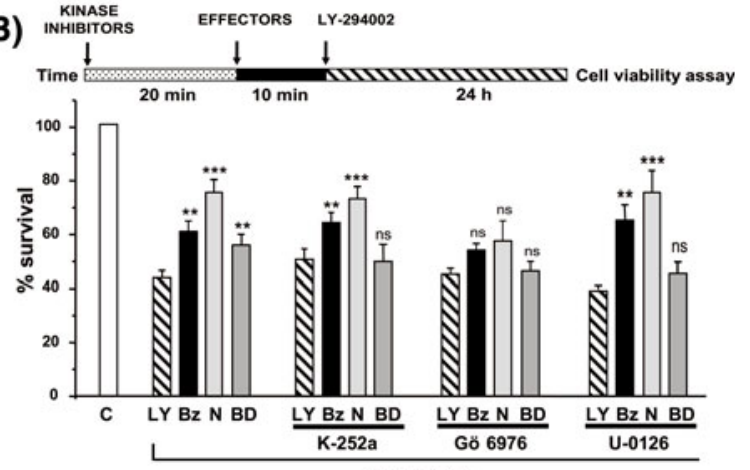

LY-294002

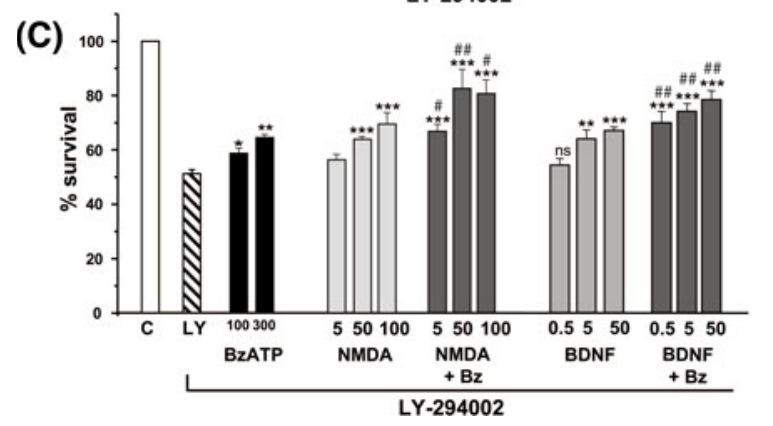

$50 \%$, being the effects obtained with BzATP and BDNF in the same range of around $30-35 \%$ (Fig. 3b). It is interesting to notice that the use of the TrkB kinase inhibitor, $\mathrm{K}-252 \mathrm{a}$, was only able to eliminate the protection elicited by BDNF, but not that induced by BzATP or NMDA, giving evidence that the neuroprotective effect mediated by BzATP and NMDA did not occur through TrkB receptor transactivation.

Importantly, we have also found that the pre-treatment with the PKC inhibitor Gö 6976 compromised the survival effects of BzATP, NMDA and specially that induced by BDNF, supporting the involvement of $\mathrm{cPKC}$ isoforms in the intracellular signalling triggered by these stimuli (Fig. 3b). Interestingly, BDNF protective effect resulted to be also dependent on MAPKs, as the MEK-1 inhibitor, U-0126, abolished the effect of BDNF without affecting responses to BzATP and NMDA.

The involvement of calcium dependent PKC isoforms in the survival effects were also corroborated by studying their calcium dependence (Supplementary Fig. 1B). Chelating extracellular calcium with EGTA/Tris completely
4 Fig. 3 Effect of BzATP, NMDA and BDNF in the regulation of neuronal survival in viability assays. The different treatments were added directly to granule neurons maintained in complete culture medium. The PI3K inhibitor, LY-294002, was added at $50 \mu \mathrm{M}$ concentration, and cell viability was analyzed $24 \mathrm{~h}$ later by the LIVE/ DEAD viability/cytotoxicity kit $\mathbf{a}$ and the MTT assay $(\mathbf{b}, \mathbf{c})$. a, b Survival effects of BzATP, NMDA and BDNF. In a granule neurons were stimulated in the absence (control) or presence of $300 \mu \mathrm{M}$ BzATP for $10 \mathrm{~min}$, before the addition of LY-294002, except in the control. The micrographs show the same field in visible (upper panels) and calcein/ethidium bromide staining (lower panels). The apoptotic nuclei are stained red, and viable cells are stained green. b Schematic representation of the experimental design followed in survival studies. Granule neurons were preincubated in the absence and presence of the kinase inhibitors: $200 \mathrm{nM} \mathrm{K-252a,} 0.5 \mu \mathrm{M}$ Gö 6976 and $10 \mu \mathrm{M} \mathrm{U}-0126$ for $20 \mathrm{~min}$ previous to the addition of the agonists, $300 \mu \mathrm{M}$ BzATP, $50 \mu \mathrm{M}$ NMDA and $50 \mathrm{ng} / \mathrm{ml}$ BDNF. After $10 \mathrm{~min}$, the PI3K inhibitor LY-294002 was added and cell viability tested $24 \mathrm{~h}$ later. c Additive survival effects of BzATP with NMDA and BDNF. The following stimulations were done before the addition of LY-294002: 100 and $300 \mu \mathrm{M}$ BzATP, 5, 50 and $100 \mu \mathrm{M}$ of NMDA, and $0.5,5$ and $50 \mathrm{ng} / \mathrm{ml}$ BDNF. The stimulations with NMDA and BDNF were carried out in the absence or presence of co-stimulation with $300 \mu \mathrm{M}$ BzATP (dark grey bars). Data are the mean $\pm \mathrm{SD}$ of at least three experiments performed in duplicate from different cultures. Data were analysed by Dunnet and Tukey tests and were statistically significant at $* * * P<0.001, * * P<0.01$ and $* P<0.05$ when treatments with LY-294002 (dashed bars in b and c) were taken as reference, and at $\# \# P<0.001$ and $\# P<0.05$ when each value was compared in the presence or absence of BzATP (dark grey bars). $L Y$ LY-294002, $B z$ BzATP, $N$ NMDA, $B D$ BDNF

abolished BzATP and NMDA protective effects. The same was obtained when the effect of BDNF was analyzed in the presence of BAPTA (Supplementary Fig. 1B).

Considering that none of the three stimuli was able to elicit a complete recovery on neuronal survival, one could anticipate that some cooperative or additive effects could be observed when these factors were acting together. As expected, co-stimulation with BzATP was able to increase the survival levels at every NMDA and BDNF concentrations (Fig. 3c), adding up their individual survival effects.

GSK3 is involved in the survival promoting effect of BzATP, NMDA and BDNF in granule neurons

The next set of experiments was conducted in order to corroborate the involvement of BzATP, NMDA and BDNF-induced GSK3 signalling in their survival promoting actions against PI3K inhibition. GSK3 phosphorylation levels were evaluated after $6 \mathrm{~h}$ exposure to the PI3K inhibitor LY-294002 $(50 \mu \mathrm{M})$. As shown in Fig. 4a, granule neurons in complete culture media exhibited high levels of basal GSK3 phosphorylation, which were almost completely lost after $6 \mathrm{~h}$ treatment with the PI3K inhibitor. Pretreatment with the survival factors BzATP, NMDA and BDNF was able to partially reverse the loss in GSK3 phosphorylation levels, being BDNF the most potent in inducing this effect. Considering that BzATP was able to 
(A)
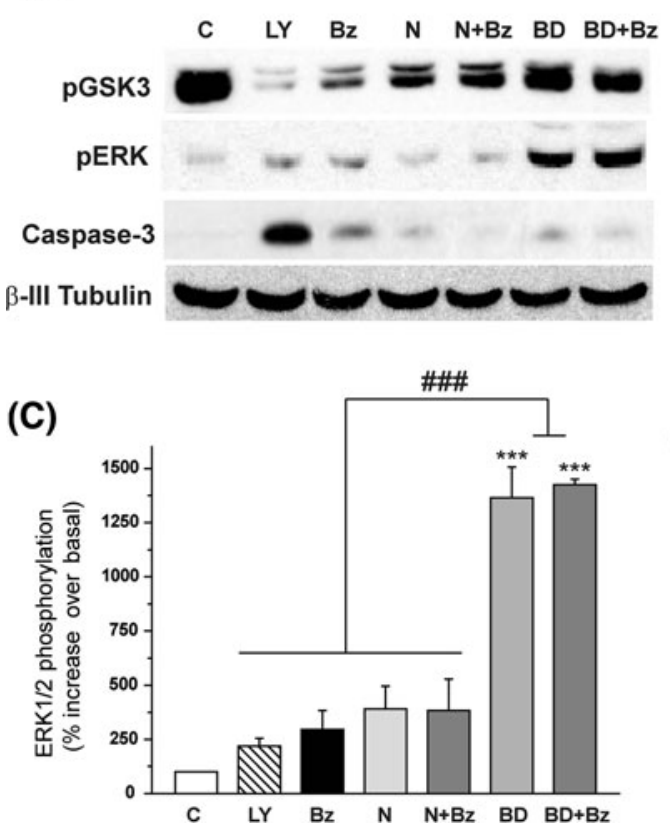

Fig. 4 Analysis of the effect of LY-294002 treatment on phosphorylation levels of GSK3 and ERK1/2, and caspase-3 activation. Granule neurons maintained in complete culture medium were treated in the presence or absence of $300 \mu \mathrm{M}$ BzATP, $50 \mu \mathrm{M}$ NMDA and $50 \mathrm{ng} / \mathrm{ml} \mathrm{BDNF}$ for $10 \mathrm{~min}$ before the addition of $50 \mu \mathrm{M} \mathrm{LY}-294002$. Cell extracts were obtained at $6 \mathrm{~h}$ incubation time with LY-294002 treatment and analysed by immunoblotting for GSK3 and ERK1/2 phosphorylation $(\mathbf{a}-\mathbf{c})$, and for the presence of the $17 \mathrm{kDa}$ active caspase-3 fragment (a, d). Histograms represent the percentage increase with respect to non-stimulated cells (100\% control value), and were obtained by normalization of densitometric values of

increase NMDA-induced GSK3 phosphorylation, a slight increase in the recovery of GSK3 phosphorylation levels was observed when these two factors were combined, which was not easy to observe with BDNF, due to its robust response (Fig. $4 \mathrm{a}, \mathrm{b}$ ). These results indicate that BzATP, as well as NMDA and BDNF, were able to rescue granule neurons against PI3K blockade by a mechanism that involves GSK3 inhibition. Interestingly, although ERK1/2 did not vary significantly after LY-294002 treatment, BDNF was the only survival factor able to induce a further increase in ERK1/2 phosphorylation levels, suggesting that MAPKs were also involved in the survival signalling mechanism mediated by BDNF (Fig. 4a, c).

The cooperation of BzATP with NMDA and BDNF was also reproduced at the level of the apoptotic inducer caspase-3. The appearance of the $17-\mathrm{kb}$ active caspase-3 fragment was clearly reversed by incubation with BzATP, NMDA and BDNF, being this reversion stronger upon the combination of BzATP with both NMDA and BDNF (Fig. 4a, d).

A series of experiments was performed in order to further confirm the intracellular signalling requirements for
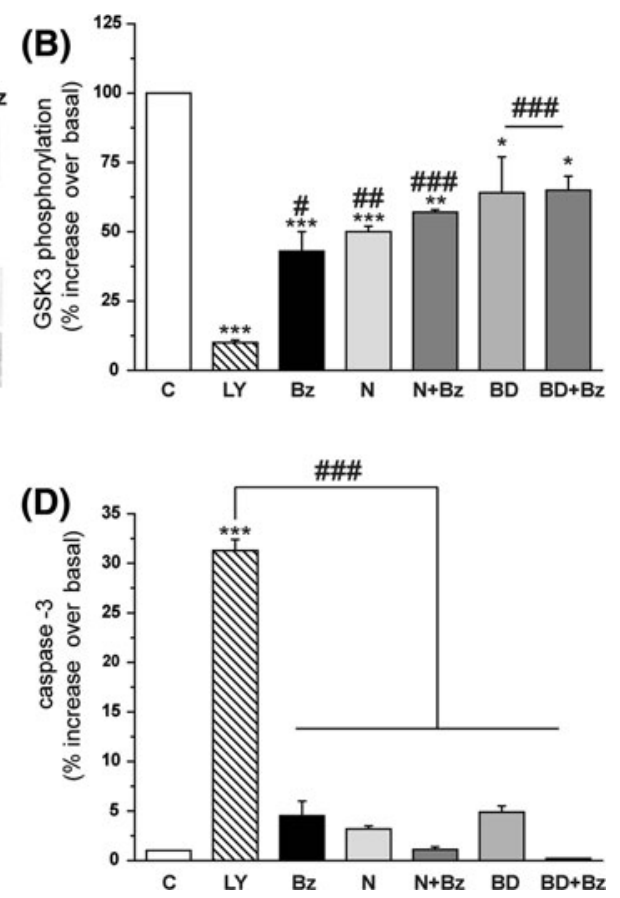

phospho-proteins and caspase-3, with respect to that obtained for $\beta$-tubulin, employed as a control charge. Values are the mean $\pm \mathrm{SD}$ of at least three experiments performed from different cultures. The blots correspond to representative experiments a. Data were analysed by Dunnet and Tukey tests and were statistically significant at $* * * P<0.001, * * P<0.01$ and $* P<0.05$ when compared to the control of untreated cells (empty bars), and at ${ }^{\# \# \#} P<0.001$, ${ }^{\# \#} P<0.01$ and ${ }^{\#} P<0.05$, when compared with LY-294002 treatment (dashed bars), as indicated in the figure. LY LY-294002, $B z$ BzATP, $N$ NMDA, $B D$ BDNF

BzATP, NMDA and BDNF involved in their survival effect against PI3K inhibition. Figure 5 show that pretreatment with the PKC inhibitor Gö 6976 did not alter the loss in GSK3 phosphorylation levels induced by the PI3K inhibitor, but totally prevented the recovery mediated by BzATP, NMDA and BDNF. Pre-treatment with the MAPK inhibitor, U-0126, was also able to diminish BDNFinduced recovery in the levels of GSK3 phosphorylation (Fig. 5c). These results taken together further corroborate that BzATP, as well as NMDA and BDNF, were able to rescue granule neurons against PI $3 \mathrm{~K}$ blockade by a mechanism that involves GSK3 inhibition. In addition, MAPKs were also involved in the survival signalling mechanism triggered by BDNF (Fig. 6).

\section{Discussion}

The present study supports the role of nucleotides as relevant physiological signals in granule neurons amplifying the neuroprotective actions of key trophic and survival stimuli in these cells, such as glutamate and BDNF. The 
(A)
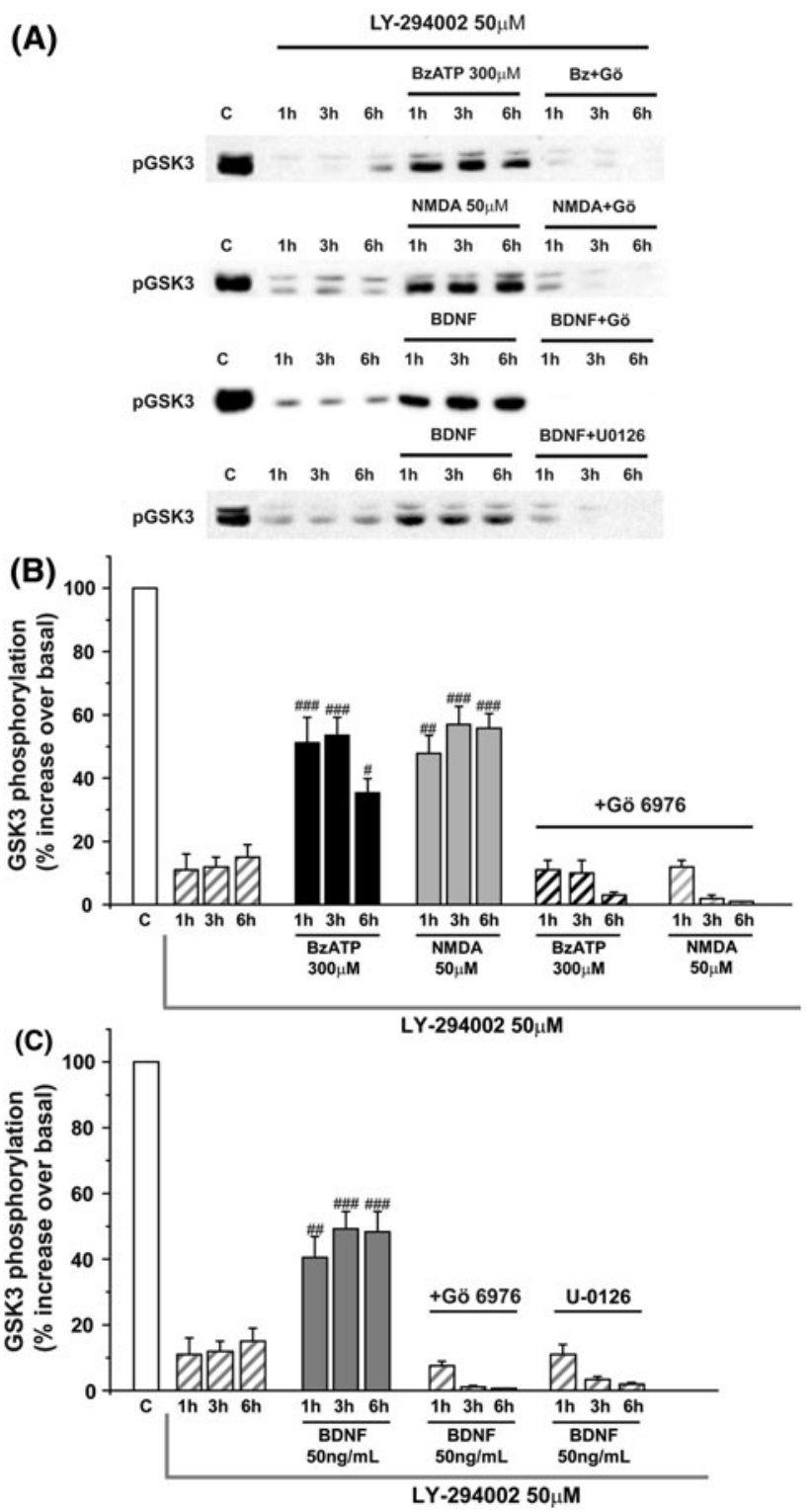

Fig. 5 Effect of PKC and MAPK inhibition on GSK3 phosphorylation levels under conditions of PI3K inhibition. Granule neurons maintained in complete culture medium were treated in the presence or absence of $300 \mu \mathrm{M}$ BzATP, $50 \mu \mathrm{M}$ NMDA and $50 \mathrm{ng} / \mathrm{ml}$ BDNF for $10 \mathrm{~min}$ before the addition of $50 \mu \mathrm{M} \mathrm{LY}-294002$. When required, the transducing inhibitors, $0.5 \mu \mathrm{M}$ Gö 6976 and $10 \mu \mathrm{M}$ U-0126 were added for $20 \mathrm{~min}$ before the addition of the three stimuli. Cell extracts were obtained at different incubation times of 1,3 and $6 \mathrm{~h}$ with LY-294002 treatment, and analysed by immunoblotting for GSK3 phosphorylation, as described before. In a the blots corresponding to representative experiments are shown. In $\mathbf{b}$ the phosphorylation values for BzATP and NMDA treatments are shown, and in $\mathbf{c}$ for BDNF. Values are the mean $\pm \mathrm{SD}$ of at least three experiments performed from different cultures. Data were analysed by Dunnet and Tukey tests, and were statistically significant at ${ }^{\# \#} P<0.001$, ${ }^{\# \#} P<0.01$ and ${ }^{\#} P<0.05$, when the values at each $L Y-294002$ incubation time were compared with respect to the treatments with Gö 6976 and U-0126. LY LY-294002, Bz. BzATP, $N$ NMDA, BD BDNF

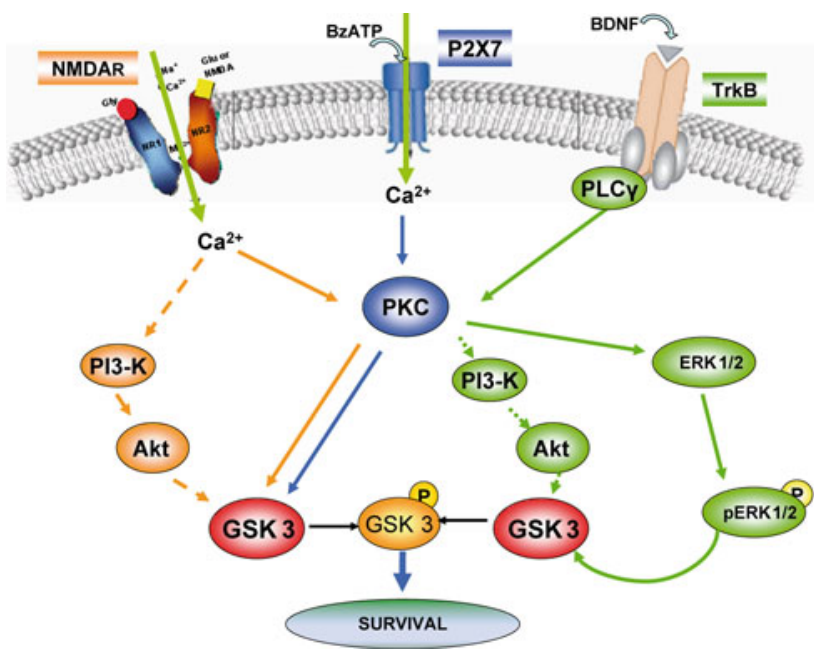

Fig. 6 Schematic diagram of P2X7, NMDA and TrkB receptors coupling to GSK3 signalling through different routes in cerebellar granule neurons. P2X7, NMDA and TrkB receptors trigger different intracellular pathways to induce GSK3 phosphorylation and elicit neuroprotection in granule neurons. P2X7 and NMDA ionotropic receptors are both coupled to the entry of extracellular calcium and share the same PKC-dependent route to provoke GSK3 phosphorylation and neuroprotection. BDNF receptor signalling is mainly coupled to the PI3K/Akt axis in a PLC/PKC-dependent manner. When $\mathrm{PI} 3 \mathrm{~K}$ is inactivated, BDNF then uses an alternative route through the activation of the ERK1/2 proteins to maintain GSK3 inhibition and elicit survival

point of convergence for the additive survival effects between the activation of $\mathrm{P} 2 \mathrm{X} 7$ receptors and NMDA or BDNF (TrkB) receptors appears to be phosphorylation of GSK3. The coupling of BzATP, NMDA and BDNF to GSK3 phosphorylation and inhibition explains the neuroprotective effects that they exert against apoptosis induced by PI3K inhibition, which mimics conditions of trophic deprivation. In addition to the well documented functions of BDNF and NMDA in granule neurons [24, 25] the present work is the first attempt to examine in depth the coupling of these factors to GSK3. Therefore, our data provide evidence that GSK3 is an important and generalized signalling target for different unrelated physiologically relevant stimuli in granule neurons including trophic factors, such as BDNF, to rapidly diffusible extracellular signals, such as ATP.

The cooperation observed for BzATP on NMDA-mediated responses occurs at the level of both GSK3 phosphorylation and neuroprotection. Although NMDA has been described to be strongly coupled to the PI3K/Akt axis in granule and cortical neurons [20, 24, 26], this does not seem to be the main pathway for GSK3. From the present results it is clear that the full effect of NMDAreceptor activation on GSK3 phosphorylation is obtained 
primarily through a PKC-dependent pathway, in the same way that BzATP acting through $\mathrm{P} 2 \mathrm{X} 7$ receptors. This is the reason why NMDA is able to rescue granule neurons from apoptosis induced by PI3K inhibition with LY-294002. Therefore, the convergence of BzATP and NMDA onto the same PKC-dependent pathway explains that their effects result additive when both signals are present at suboptimal concentrations (Fig. 6). The sum of effects shown for BzATP and NMDA in granule neurons indicates a new and interesting example of interaction between P2X7 and NMDA receptors. The different approaches used revealed that calcium-dependent PKC isoforms were responsible for the GSK3 phosphorylation and the neuroprotective effects displayed by the three effectors, which could corresponded to $\mathrm{PKC} \alpha$ and $\mathrm{PKC} \beta$ subtypes [23].

The cooperation between BzATP and BDNF was clearly assessed at the level of the survival promoting effect against the sustained PI3K inhibition. BDNF neuroprotective effect depends on different signalling mechanisms that involve both GSK3 and ERK proteins, and these effects are additive to those displayed by BzATP, even when both factors are present at maximal concentrations. Thus, they will be acting on different intracellular targets to stop the activation of the apoptotic machinery. BDNF has been reported to activate transcription factors, such as CREB and $\mathrm{NF} \kappa \mathrm{B}$, in an ERK-dependent fashion, and to increase the expression of anti-apoptotic genes of the $b c l-2$ family [24]. In granule neurons, the protein Bax has been described to be phosphorylated by GSK3, and this could be one possible target for $\mathrm{P} 2 \mathrm{X} 7$ receptors [15].

Another interesting point to examine in more detail is the signalling mechanism elicited by BDNF in granule neurons. Our results support a key role for $\mathrm{PKC}$ as a common upstream effector of both GSK3 and ERK pathways activated by BDNF (Fig. 6). This is in agreement to that reported for TrkB receptor activation in cortical and granule neurons, which is primarily coupled to PLC $\gamma$ phosphorylation and the subsequent activation of $\mathrm{cPKC}$. From cPKC, two pathways are separated, leading either to ERK activation through the intermediate Ras, or to activation of the PI3K/Akt axis [27]. It appears that these branching routes are independent, as GSK3 phosphorylation, but not ERK1/2, is completely dependent on the PI3K/Akt axis. Furthermore, GSK3 phosphorylation mediated by BDNF seems to be unaffected by MAPK inhibition. Nevertheless, the survival studies carried out in the present work provide evidence that some degree of cross-talk is taking place, and that it can be triggered when changing the environmental conditions. Indeed, BDNFstimulated GSK3 phosphorylation and neuroprotection becomes MAPK-dependent only when the PI3K/Akt axis is impaired. Therefore this is pointing to ERK proteins as an alternative route reaching to GSK3. In agreement with our present results, studies performed in cortical neurons have reported that both NMDA and BDNF can alternatively use the ERK route as the survival mechanism to compensate for the loss of PI3K activity. Although in this case, ERK-mediated inhibition of GSK3 catalytic activity was occurring through a mechanism different to GSK3 phosphorylation events and that could involve the complex formation between the two proteins [28]. This is different to that seen in our results, as BDNF-mediated neuroprotection involved GSK3 phosphorylation in an ERKdependent way. In this respect, $\mathrm{p} 90^{\text {rsk }}$ (RSKs) has been described as the candidate upstream kinase for GSK3 phosphorylation in Ser residues after the assembly and priming of GSK3 with ERK1/2 proteins [29]. Therefore, it cannot be excluded that a similar mechanism of interplay between GSK3 and ERK proteins could be taking place in granule neurons.

The versatility of BDNF as a survival factor is further supported by other examples in which this neurotrophin is capable of discriminating between different signalling routes depending on the type of noxious stimuli. When all the intracellular cascades are fully functional, BDNF preferentially uses the PI3K/Akt route to protect against trophic factor deprivation, and alternatively uses ERK signalling to protect against DNA damaging agents [25].

Finally, it is interesting to consider and emphasize the possible physiological implications of the cooperation described in the present study between different kinds of extracellular signals, such as ATP with glutamate and BDNF. This kind of interactions could have a relevant special meaning taking into account that these factors are part of a reinforcement survival mechanism that operates in granule and hippocampal neurons [24]. Many of the NMDA protective actions are attributed to BDNF secretion, which then activates TrkB receptors on this neuron itself or on surrounding neurons to elicit survival responses. Indeed, the intracellular signals activated by NMDA, such as ERK and Akt, are partially dependent on TrkB activation, as also observed for GSK3 signalling in the present study [30]. Although many examples exist in the literature concerning $\mathrm{P} 2 \mathrm{Y}$ nucleotide receptors interacting with neurotrophins [31], our results with $\mathrm{P} 2 \mathrm{X} 7$ receptors in granule neurons give evidence that releasing mechanisms or TrkB transactivating effects are not contributing to the observed BzATP-mediated neuroprotection. Nevertheless, it cannot be excluded that this type of cross-mechanisms could take place in more physiological conditions, taking into account that some precedents are found in recent interesting works regarding the effect of nucleotide receptors in both $\mathrm{BDNF}$ expression and release. In relation to this, $\mathrm{P} 2 \mathrm{X} 7$ receptors are responsible for $\mathrm{BDNF}$ release from Schwann cells, which display neuroprotective actions on neighbouring vestibular neurons [32]. In activated 
microglia, $\mathrm{P} 2 \mathrm{X} 4$ is up-regulated and promotes BDNF release in spinal cord neurons contributing to pain signalling [33]. In addition, ATP is reported to induce BDNF gene expression in cortical astrocytes acting through a $\mathrm{P}_{2} \mathrm{Y}_{1}$ receptor, an effect involving CaMKII activation and CREB-induced transcription [34].

As observed in the present studies, there is growing evidence that ATP and nucleotides are becoming important priming molecules able to interact with other extracellular signals to amplify or modulate their signalling and survival actions in neurones [35]. These actions could be of special relevance under conditions in which trophic support is being compromised, as for example, in some inflammatory and neurodegenerative insults. Whether nucleotides can participate in the NMDA-BDNF loop in granule neurons is the aim of future work and could open a new dimension in the potential protective role of nucleotides in the nervous system.

Acknowledgments We thank Dr. Charles Hoyle for his criticisms and English corrections of the manuscript. Verónica Morente is a fellowship of MECD and Felipe Ortega has a post-doctoral position. This works was supported by research grants from the Spanish Ministry of Education and Science BFU2008-02699, the Fundación Marcelino Botín, Comunidad de Madrid (S-SAL-0253-2006) and Consolider-Ingenio-CSD2008-00005 ("The Spanish ion channel initiative").

Open Access This article is distributed under the terms of the Creative Commons Attribution Noncommercial License which permits any noncommercial use, distribution, and reproduction in any medium, provided the original author(s) and source are credited.

\section{References}

1. Contestabile A (2002) Cerebellar granule cells as a model to study mechanisms of neuronal apoptosis or survival in vivo and in vitro. Cerebellum 1:41-55

2. Miller TM, Tansey MG, Johnson EM Jr, Creedon DJ (1997) Inhibition of phosphatidylinositol 3-kinase activity blocks depolarization- and insulin-like growth factor I-mediated survival of cerebellar granule cells. J Biol Chem 272:9847-9853

3. Hervás C, Pérez-Sen R, Miras-Portugal MT (2003) Coexpression of functional P2X and P2Y nucleotide receptors in single cerebellar granule cells. J Neurosci Res 73:384-399

4. Amadio S, D'Ambrosi N, Cavaliere F, Murra B, Sancesario G, Bernardi G, Burnstock G, Volonte C (2002) P2 receptor modulation and cytotoxic function in cultured CNS neurons. Neuropharmacology 42:489-501

5. North RA (2002) Molecular physiology of P2X receptors. Physiol Rev 82:1013-1067

6. Abbracchio MP, Burnstock G, Boeynaems JM, Barnard EA, Boyer JL, Kennedy C, Knight GE, Fumagalli M, Gachet C, Jacobson KA, Weisman GA (2006) International Union of Pharmacology LVIII: update on the P2Y G protein-coupled nucleotide receptors: from molecular mechanisms and pathophysiology to therapy. Pharmacol Rev 58:281-341
7. Hervás C, Pérez-Sen R, Miras-Portugal MT (2005) Presence of diverse functional $\mathrm{P} 2 \mathrm{X}$ receptors in rat cerebellar synaptic terminals. Biochem Pharmacol 70:770-785

8. León D, Hervás C, Miras-Portugal MT (2006) P2Y1 and P2X7 receptors induce calcium/calmodulin-dependent protein kinase II phosphorylation in cerebellar granule neurons. Eur J Neurosci 23:2999-3013

9. Jope RS, Johnson GV (2004) The glamour and gloom of glycogen synthase kinase-3. Trends Biochem Sci 29:95-102

10. Ding VW, Chen RH, McCormick F (2000) Differential regulation of glycogen synthase kinase 3beta by insulin and Wnt signaling. J Biol Chem 275:32475-32481

11. Ortega F, Pérez-Sen R, Miras-Portugal MT (2008) Gi-coupled P2Y-ADP receptor mediates GSK-3 phosphorylation and betacatenin nuclear translocation in granule neurons. J Neurochem 104:62-73

12. Ortega F, Perez-Sen R, Delicado EG, Miras-Portugal MT (2009) P2X7 nucleotide receptor is coupled to GSK-3 inhibition and neuroprotection in cerebellar granule neurons. Neurotox Res 15:193-204

13. Beurel E, Jope RS (2006) The paradoxical pro- and anti-apoptotic actions of GSK3 in the intrinsic and extrinsic apoptosis signaling pathways. Prog Neurobiol 79:173-189

14. Crowder RJ, Freeman RS (2000) Glycogen synthase kinase-3 beta activity is critical for neuronal death caused by inhibiting phosphatidylinositol 3-kinase or Akt but not for death caused by nerve growth factor withdrawal. J Biol Chem 275:34266-34271

15. Linseman DA, Butts BD, Precht TA, Phelps RA, Le SS, Laessig TA, Bouchard RJ, Florez-McClure ML, Heidenreich KA (2004) Glycogen synthase kinase-3beta phosphorylates Bax and promotes its mitochondrial localization during neuronal apoptosis. J Neurosci 24:9993-10002

16. Hongisto V, Smeds N, Brecht S, Herdegen T, Courtney MJ, Coffey ET (2003) Lithium blocks the c-Jun stress response and protects neurons via its action on glycogen synthase kinase 3 . Mol Cell Biol 23:6027-6036

17. Chin PC, Majdzadeh N, D'Mello SR (2005) Inhibition of GSK3beta is a common event in neuroprotection by different survival factors. Brain Res Mol Brain Res 137:193-201

18. Pap M, Cooper GM (1998) Role of glycogen synthase kinase-3 in the phosphatidylinositol 3-Kinase/Akt cell survival pathway. J Biol Chem 273:19929-19932

19. Hetman M, Cavanaugh JE, Kimelman D, Xia Z (2000) Role of glycogen synthase kinase-3beta in neuronal apoptosis induced by trophic withdrawal. J Neurosci 20:2567-2574

20. Hetman M, Kharebava G (2006) Survival signaling pathways activated by NMDA receptors. Curr Top Med Chem 6:787-799

21. Pons S, Trejo JL, Martinez-Morales JR, Marti E (2001) Vitronectin regulates Sonic hedgehog activity during cerebellum development through CREB phosphorylation. Development 128:1481-1492

22. Johnson-Farley NN, Travkina T, Cowen DS (2006) Cumulative activation of akt and consequent inhibition of glycogen synthase kinase-3 by brain-derived neurotrophic factor and insulin-like growth factor-1 in cultured hippocampal neurons. J Pharmacol Exp Ther 316:1062-1069

23. Popp RL, Velasquez O, Bland J, Hughes P (2006) Characterization of protein kinase $\mathrm{C}$ isoforms in primary cultured cerebellar granule cells. Brain Res 1083:70-84

24. Jiang X, Zhu D, Okagaki P, Lipsky R, Wu X, Banaudha K, Mearow K, Strauss KI, Marini AM (2003) N-methyl-D-aspartate and TrkB receptor activation in cerebellar granule cells: an in vitro model of preconditioning to stimulate intrinsic survival pathways in neurons. Ann N Y Acad Sci 993:134-145 discussion $159-60$ 
25. Hetman M, Kanning K, Cavanaugh JE, Xia Z (1999) Neuroprotection by brain-derived neurotrophic factor is mediated by extracellular signal-regulated kinase and phosphatidylinositol 3-kinase. J Biol Chem 274:22569-22580

26. Zhang FX, Rubin R, Rooney TA (1998) N-Methyl-D-aspartate inhibits apoptosis through activation of phosphatidylinositol 3-kinase in cerebellar granule neurons. A role for insulin receptor substrate-1 in the neurotrophic action of $N$-methyl-D-aspartate and its inhibition by ethanol. J Biol Chem 273:26596-26602

27. Zirrgiebel U, Ohga Y, Carter B, Berninger B, Inagaki N, Thoenen H, Lindholm D (1995) Characterization of TrkB receptor-mediated signaling pathways in rat cerebellar granule neurons: involvement of protein kinase $\mathrm{C}$ in neuronal survival. J Neurochem 65:2241-2250

28. Hetman M, Hsuan SL, Habas A, Higgins MJ, Xia Z (2002) ERK1/2 antagonizes glycogen synthase kinase-3beta-induced apoptosis in cortical neurons. J Biol Chem 277:49577-49584

29. Ding Q, Xia W, Liu JC, Yang JY, Lee DF, Xia J, Bartholomeusz G, Li Y, Pan Y, Li Z, Bargou RC, Qin J, Lai CC, Tsai FJ, Tsai $\mathrm{CH}$, Hung MC (2005) Erk associates with and primes GSK-3beta for its inactivation resulting in upregulation of beta-catenin. Mol Cell 19:159-170

30. Marini AM, Rabin SJ, Lipsky RH, Mocchetti I (1998) Activitydependent release of brain-derived neurotrophic factor underlies the neuroprotective effect of $N$-methyl-D-aspartate. J Biol Chem 273:29394-29399

31. Neary JT, Kang Y, Shi YF (2004) Signaling from nucleotide receptors to protein kinase cascades in astrocytes. Neurochem Res 29:2037-2042

32. Verderio C, Bianco F, Blanchard MP, Bergami M, Canossa M, Scarfone E, Matteoli M (2006) Cross talk between vestibular neurons and Schwann cells mediates BDNF release and neuronal regeneration. Brain Cell Biol 35:187-201

33. Ulmann L, Hatcher JP, Hughes JP, Chaumont S, Green PJ, Conquet F, Buell GN, Reeve AJ, Chessell IP, Rassendren F (2008) Up-regulation of P2X4 receptors in spinal microglia after peripheral nerve injury mediates BDNF release and neuropathic pain. J Neurosci 28:11263-11268

34. Takasaki I, Takarada S, Tatsumi S, Azegami A, Yasuda M, Fukuchi M, Tabuchi A, Kondo T, Tabuchi Y, Tsuda M (2008) Extracellular adenosine $5^{\prime}$-triphosphate elicits the expression of brain-derived neurotrophic factor exon IV mRNA in rat astrocytes. Glia 56:1369-1379

35. D'Ambrosi N, Murra B, Cavaliere F, Amadio S, Bernardi G, Burnstock G, Volonte C (2001) Interaction between ATP and nerve growth factor signalling in the survival and neuritic outgrowth from PC12 cells. Neuroscience 108:527-534 